\title{
AVALIAÇÃO IN VITRO DO FATOR DE PROTEÇÃO SOLAR A PARTIR DE FOTOPROTETORES MANIPULADOS CONTENDO AGENTES ATIVOS ANTIENVELHECIMENTO
}

\author{
Beatriz Lino Alves ${ }^{1}$, Jacqueline Alves Pereira ${ }^{1}$, Rosângela Cristóvão Ferreira ${ }^{1}$, Ana Julia Pereira Santinho \\ Gomes $^{2}$ \\ ${ }^{1}$ Curso de Farmácia da Universidade do Oeste Paulista, Presidente Prudente, SP; ${ }^{2}$ Faculdade de Farmácia, Universidade Federal de \\ São João Del-Rei, Divinópolis, MG. ajpsant@yahoo.com
}

\section{RESUMO}

Questões críticas foram levantadas envolvendo interações potenciais entre os componentes do filtro solar e os componentes de agentes ativos antienvelhecimento (AA). O objetivo foi avaliar fotoprotetores contendo diferentes agentes AA, com ou sem fragrância, que foram submetidos à uma análise macroscópica, centrifugação, determinação de $\mathrm{pH}$ e o fator de proteção solar (FPS) in vitro. Todas as preparações apresentaram estabilidade física. No entanto, as formulações contendo Deanol ${ }^{\circledR}$ apresentaram $\mathrm{o} \mathrm{pH}$ significativamente mais baixo do que os outros fotoprotetores, independentemente da fragrância, indicando que a estabilidade destas formulações pode ser menor. Quanto à variação do FPS in vitro não foi encontrada diferença significativa após a adição do agente de AA em fotoprotetores. No entanto, os filtros solares contendo Enteline ${ }^{\circledR}$ apresentaram o FPS significativamente maior nas formulações sem fragrância. Em conclusão, as preparações estudadas apresentaram estabilidade física e o intervalo de estabilidade apresentado do $\mathrm{pH}$ em conformidade com a necessidade de filtros solares contendo compostos

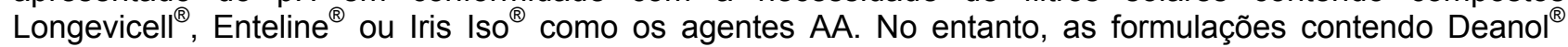
devem ser ajustadas para a o intervalo de $\mathrm{pH}(6,0-7,0)$, independentemente da fragrância. Além disso, a fragrância usada em fotoprotetores Enteline ${ }^{\circledR}$ avaliados neste trabalho pode diminuir significativamente o FPS na preparação e devem ser evitados a fim de garantir a segurança e eficácia dos protetores solares associadas aos agentes AA.

Palavras-chave: fator de proteção solar, FPS in vitro, proteção solar, agentes antienvelhecimento, estabilidade física.

\section{IN VITRO EVALUATION OF SUN PROTECTION FACTOR FROM COMPOUNDED SUNSCREENS CONTAINING ANTIAGING ACTIVE AGENTS}

\begin{abstract}
Critical issues have taken place involving potential interactions between sunscreen components and the components of antiaging active (AA) agents. Critical issues have taken place involving potential interactions between sunscreen components and the components of antiaging active (AA) agents. The aim was to evaluate compounded sunscreens containing different AA agents with or without fragrance, which were submitted to macroscopic analysis, centrifugation, $\mathrm{pH}$ determination and in vitro sun protection factor (SPF). All preparations presented physical stability. However, formulations containing Deanol ${ }^{\circledR}$ exhibited $\mathrm{pH}$ significantly lower than the others independent to the fragrance, indicating that the stability of these formulations may be lower. As regards in vitro SPF variation no significantly difference was found after adding to AA agent into compounded sunscreens. However, sunscreens containing Enteline ${ }^{\circledR}$ presented SPF significantly higher in formulations with no fragrance. In conclusion, the preparations studied showed physical stability and the $\mathrm{pH}$ range of stability presented in accordance with the need for compounded sunscreens containing Longevicell ${ }^{\circledR}$, Enteline ${ }^{\circledR}$ or Iris Iso ${ }^{\circledR}$ as AA agents. Nevertheless, formulations containing Deanol ${ }^{\circledR}$ should be adjusted to $\mathrm{pH}$ range $(6.0-7.0)$, regardless of the fragrance. Furthermore, the fragrance used in compounded sunscreens containing Enteline ${ }^{\circledR}$ assessed in this work can significantly decrease the SPF in the preparation and should be avoided in order to ensure the safety and efficacy of sunscreens associated with AA agents.
\end{abstract}

Keywords: sun protection factor, in vitro SPF, sunscreens, antiaging agents, physical stability. 


\section{INTRODUÇÃO}

Os requisitos gerais que os protetores solares modernos devem cumprir são capacidade de proteger a pele contra as radiações UVB e UVA, fotoestabilidade e resistência à água (KRUTMANN; YAROSH, 2006).

Entre os métodos utilizados para determinar a eficácia dos filtros solares está à análise espectrofotométrica, onde o FPS, estimado por espectrofotometria, é um número que mede o filtro solar de acordo com a altura, largura e localização da sua curva de absorção no espectro ultravioleta (MANSUR et al., 1986).

A profilaxia do envelhecimento precoce da pele tem assumido uma importância crescente na população em geral e o componente essencial das estratégias de prevenção é a fotoproteção da pele (KRUTMANN; YAROSH, 2006; KRUTMANN; GILCHREST, 2006).

Questões críticas foram levantadas envolvendo interações potenciais entre os componentes do filtro solar e os componentes de agentes ativos antienvelhecimento. Isso ocorre desde que os filtros UV químicos possam reduzir a eficácia de hidratantes por exemplo. Concomitantemente, os agentes hidratantes poderiam interromper a película protetora dos filtros solares. Os antioxidantes têm sido conhecidos por exercer um efeito protetor sobre os ingredientes de filtros solares, melhorando a sua fotoestabilidade. Estas questões apresentam desafios e oportunidades na formulação de produtos multinacionais inovadores (STANFIELD, 2003).

Em paralelo, é importante considerar o caráter aquoso, oleoso ou emulsivo das bases nas quais os filtros UV são incorporados, principalmente porque essas substâncias têm diferentes solubilidades (FONSECA; PRISTA, 2000). Isto pode interferir conseqüentemente nas propriedades absortivas de um determinado filtro UV. A associação de filtros UV químicos e agentes ativos antienvelhecimento deve ser cautelosa, pois pode ocorrer a diminuição do FPS pretendido, o que pode causar aumento do risco de câncer de pele (KRUTMANN; YAROSH, 2006; BORGUETTI; KNORST, 2006).

O objetivo deste trabalho foi avaliar parâmetros qualitativos e quantitativos de filtros solares manipulados contendo diferentes agentes ativos antienvelhecimento, sobretudo o fator de proteção solar (FPS) in vitro.

\section{MÉTODOS}

\section{Material}

Deanol $^{\circledR}$ (dimetil amino etanol, DMAE) / DMAE Fluido ou DMAE Pidolato (JuK Kraeber Gmbh \& Co, Purifarma, Brasil), Longevicell ${ }^{\circledR}$ (Silab, Galena, Brasil), Enteline ${ }^{\circledR} 2$ (Secma Biotecnologias, Deg, Brasil) e Iris Iso ${ }^{\circledR}$ (Silab, Galena, Brasil) foram utilizados como agentes ativos antienvelhecimento. Fragrância ALL31024 (All Chemistry, Brasil). Todos os outros reagentes foram de grau analítico e utilizado conforme recebidos.

\section{Formulação do protetor solar}

Lotes $(n=3)$ de cada filtro solar manipulado $(20 \mathrm{~g})$ foram preparados a cada 15 dias e doados por uma Farmácia de Manipulação em Presidente Prudente, SP, Brasil, os quais eram compostos por filtros UV químicos incorporados em uma emulsão O / A com FPS 30 desejado.

Quatro agentes ativos antienvelhecimento foram individualmente adicionados aos fotoprotetores manipulados, os quais foram divididos em duas categorias, de acordo com a presença da fragrância. A Tabela 1 apresenta estas categorias de produtos submetidos à análise e seus respectivos grupos. 
Tabela 1. Formulações dos filtros solares manipulados analisados.

\begin{tabular}{|c|c|}
\hline Formulação & $\begin{array}{l}\text { Componentes das preparações } \\
\text { sem fragrância }\end{array}$ \\
\hline 1 & $\begin{array}{l}\text { Protetor solar FPS } 30 \text { (controle } \\
\text { positivo, sem fragrância) }\end{array}$ \\
\hline 2 & $\begin{array}{l}\text { Protetor solar FPS } 30 \text {, acrescido } \\
\text { de } 3 \% \text { Deanol }{ }^{\circledR} / \text { DMAE Fluido }\end{array}$ \\
\hline 3 & $\begin{array}{l}\text { Protetor solar FPS } 30 \text {, acrescido } \\
\text { de } 1 \% \text { Longevicell }^{\circledR}\end{array}$ \\
\hline 4 & $\begin{array}{l}\text { Protetor solar FPS } 30 \text {, acrescido } \\
\text { de } 2 \% \text { Enteline }{ }^{\circledR} 2\end{array}$ \\
\hline \multirow[t]{2}{*}{5} & $\begin{array}{l}\text { Protetor solar FPS } 30 \text {, acrescido } \\
\text { de } 3 \% \text { Iris Iso }{ }^{\circledR}\end{array}$ \\
\hline & $\begin{array}{l}\text { Componentes das preparações } \\
\text { contendo fragrância }\end{array}$ \\
\hline 6 & $\begin{array}{l}\text { Protetor solar FPS } 30 \text { (controle } \\
\text { positivo contendo fragrância) }\end{array}$ \\
\hline 7 & $\begin{array}{l}\text { Protetor solar FPS } 30 \text {, acrescido } \\
\text { de } 3 \% \text { Deanol }^{\circledR} / \text { DMAE Fluído }\end{array}$ \\
\hline 8 & $\begin{array}{l}\text { Protetor solar FPS } 30 \text {, acrescido } \\
\text { de } 1 \% \text { Longevicell }^{\circledR}\end{array}$ \\
\hline 9 & $\begin{array}{l}\text { Protetor solar FPS } 30 \text {, acrescido } \\
\text { de } 2 \% \text { Enteline }{ }^{\circledR} 2\end{array}$ \\
\hline 10 & $\begin{array}{l}\text { Protetor solar FPS } 30 \text {, acrescido } \\
\text { de } 3 \% \text { Iris Iso }{ }^{\circledR}\end{array}$ \\
\hline
\end{tabular}

Todas as preparações foram mantidas em temperatura ambiente controlada e analisadas em triplicara no seu prazo de validade.

\section{Análise macroscópica}

A análise macroscópica (visual) foi realizada com o acompanhamento características sensoriais e uniformidade das formulações, a fim de identificar prováveis processos de instabilidade. Este procedimento foi realizado com base em Vieira et al. (2009) como segue: 1) Aspecto: NS - Não há separação da fase oleosa; S - separação da fase oleosa. 2) Cor: N - Normal; M - Modificado; IM - intensamente modificado. 3) Odor: N - Normal; M - Modificado; IM intensamente modificado 4) Espalhabilidade e toque: A - toque agradável, fácil aplicação na pele; $\mathrm{D}$ - toque desagradável, pegajoso, relativa dificuldade para espalhar na pele; MD - toque muito desagradável, muito pegajoso, compromete a aplicação na pele.

\section{Centrifugação}

A estabilidade física, por exemplo, cremagem ou sedimentação, das formulações foram testadas através da realização de centrifugação (Olidef CZ-CD 4000) a 3000 rpm durante 30 minutos usando $5 \mathrm{~g}$ de cada formulação (TADROS, 1992; BRASIL, 2004).

\section{Determinação do pH}

De acordo com Souza (2004), o pH de uma preparação fotoprotetora é importante para a estabilidade do filtro UV empregado e deve estar entre 6.0 e 7.0. Para tanto, uma solução aquosa de cada formulação (10\% p/ V) foi preparada. Em seguida, as amostras foram aquecidas a $70^{\circ} \mathrm{C}$, resfriado a temperatura ambiente e filtradas em algodão. Finalmente, o eletrodo foi imerso no filtrado para a realização da leitura, utilizando um pHmetro Quimis Q400A (GIL, 2007).

\section{Determinação in vitro do FPS}

$O$ FPS in vitro dos produtos foi determinado de acordo com o método descrito por Mansur (1986) utilizando espectrofotômetro Shimadzu UV-1601PC. As formulações foram diluídas em álcool isopropílico na concentração final de $0.2 \mu \mathrm{L} / \mathrm{mL}$ da emulsão para as leituras espectrofotométricas. Para tanto, $0,100 \mathrm{~g}$ do fotoprotetor foi acuradamente pesada, transferida para um balão de $100 \mathrm{ml}$ e adicionado o volume de álcool isopropílico. Em seguida, diluições seriadas foram realizadas a fim de obter as amostras na concentração para leituras, que foram realizadas em $290-320 \mathrm{~nm}$, com intervalos de $5 \mathrm{~nm}$. As análises foram realizadas em triplicata e o FPS foi calculado através da equação 1: 


$$
F P S=F C \times \sum_{290}^{320} \times E E(\lambda) \times I(\lambda) \times A b s(\lambda)
$$

(Equação 1)

Onde FC = 10 (fator de correção), determinado de acordo com dois filtros UV FPS conhecido de tal maneira que um creme contendo $8 \%$ de homossalato resultou em FPS 4; EE $(\lambda)=$ efeito eritematogênico de radiação de onda $\lambda ;$ I $(\lambda)$ $=$ intensidade da luz solar no comprimento de onda $\lambda$; Abs $(\lambda)=$ valor de absorbância espectrofotométrica de comprimento de onda $\lambda$ por uma solução de preparação. Os valores de EE $(\lambda)$ e I $(\lambda)$ usados podem ser encontrados na Tabela 2.

\section{Análise estatística}

Os resultados são dados como média aritmética \pm 0 valor do desvio padrão $(X \pm D P)$. Comparações estatísticas foram feitas através de análise de variância (one-way ANOVA) seguidas do pós-teste de Tukey HSD (honest significance difference) com $\mathrm{P}<0,05$ como nível mínimo de significância e 95,0\% dos intervalos de confiança.

Tabela 2. Valores empregados na determinação do FPS por espectrofotometria.

\begin{tabular}{cc}
\hline Comprimento de onda $(\mathbf{n m})$ & $\mathrm{EE} \times \mathrm{I}$ \\
\hline 290 & 0,0150 \\
295 & 0,0817 \\
300 & 0,2874 \\
305 & 0,3278 \\
310 & 0,1864 \\
315 & 0,839 \\
320 & 0,0180 \\
& 1,0000 \\
\hline
\end{tabular}

Fonte: Santos et al. (1999).

\section{RESULTADOS}

Após o terceiro lote de formulações, cada avaliação foi realizada em triplicata. Os resultados qualitativos e quantitativos deste trabalho estão descritos na Tabela 3.

\section{DISCUSSÃO}

Os resultados da Tabela 3 mostram que todas as preparações apresentaram estabilidade física suficiente uma vez que não foi observada alterações de cor, odor e aspecto, mesmo após a centrifugação. De acordo com Sanfeld e Steinchen (2008) o cosmético, com processos de sedimentação e separação de dispersão líquidolíquido desempenhou um papel importante em diversas aplicações industriais, enquanto que as gotas de emulsão foram comprimidas por uma pressão externa por meio de centrifugação. Devido à diferença de densidade entre as fases de uma rede de força que se desenvolve dentro do sistema, em resposta à força aplicada, pode ser assegurada a qualidade das emulsões.

No que diz respeito ao $\mathrm{pH}$, é importante considerar que a faixa de $\mathrm{pH}$ de máxima estabilidade, para cada agente antienvelhecimento ativo é 4,0-7,5 para Deanol $^{\circledR}$, DMAE Fluido (formulações 2 e 7); 2,0-7,0 para Longevicell $^{\circledR}$ (Formulações 3 e 8 ); 5,5-6,5 para Enteline ${ }^{\circledR} 2$ (Formulações 4 e 9) e 5,0-10,0 por Iris Iso $\circledR^{(F o r m u l a c ̧ o ̃ e s ~} 5$ e 10) (SOUZA, 2004; SOUZA, 2005; Longevicell ${ }^{\circledR}$, 2010). 
Tabela 3. Avaliação das características organolépticas, os valores de pH e fator de proteção solar FPS in vitro com componentes de agentes ativos antienvelhecimento a ser preparados sem fragrância (Formulações 1-5) e preparações contendo fragrância (Formulações 6-10).

\begin{tabular}{cccccccc}
\hline & \multicolumn{6}{c}{ Parâmetros avaliados } \\
\cline { 2 - 8 } Formulação & Aspecto & Cor & Odor & $\begin{array}{c}\text { Espalhabilidade } \\
\text { toque }\end{array}$ & $\mathrm{pH}$ & FPS in vitro \\
\hline $\mathbf{1}$ & $\mathrm{NS}$ & $\mathrm{N}$ & $\mathrm{N}$ & $\mathrm{A}$ & $6.57 \pm 0.14$ & $35.52 \pm 4.59$ \\
$\mathbf{2}$ & $\mathrm{NS}$ & $\mathrm{N}$ & $\mathrm{N}$ & $\mathrm{A}$ & $3.61 \pm 0.06^{\dagger}$ & $33.01 \pm 5.65$ \\
$\mathbf{3}$ & $\mathrm{NS}$ & $\mathrm{N}$ & $\mathrm{N}$ & $\mathrm{A}$ & $6.63 \pm 0.25$ & $31.05 \pm 3.28$ \\
$\mathbf{4}$ & $\mathrm{NS}$ & $\mathrm{N}$ & $\mathrm{N}$ & $\mathrm{A}$ & $6.45 \pm 0.17$ & $34.49 \pm 6.31^{\ddagger}$ \\
$\mathbf{5}$ & $\mathrm{NS}$ & $\mathrm{N}$ & $\mathrm{N}$ & $\mathrm{A}$ & $6.49 \pm 0.20$ & $31.24 \pm 2.49$ \\
$\mathbf{6}$ & $\mathrm{NS}$ & $\mathrm{N}$ & $\mathrm{N}$ & $\mathrm{A}$ & $6.53 \pm 0.19$ & $29.40 \pm 1.39$ \\
$\mathbf{7}$ & $\mathrm{NS}$ & $\mathrm{N}$ & $\mathrm{N}$ & $\mathrm{A}$ & $3.61 \pm 0.04{ }^{\dagger}$ & $28.85 \pm 4.41$ \\
$\mathbf{8}$ & $\mathrm{NS}$ & $\mathrm{N}$ & $\mathrm{N}$ & $\mathrm{A}$ & $6.46 \pm 0.32$ & $26.77 \pm 1.66$ \\
$\mathbf{9}$ & $\mathrm{NS}$ & $\mathrm{N}$ & $\mathrm{N}$ & $\mathrm{A}$ & $6.51 \pm 0.28$ & $26.58 \pm 3.09$ \\
$\mathbf{1 0}$ & $\mathrm{NS}$ & $\mathrm{N}$ & $\mathrm{N}$ & $\mathrm{A}$ & $6.52 \pm 0.35$ & $26.07 \pm 1.67$
\end{tabular}

Legenda: Aspecto: NS - Não há separação da fase oleosa; S - separação da fase oleosa. Cor: N - Normal; M - Modificado; IM intensamente modificado. Odor: N - Normal; M - Modificado; IM - intensamente modificado. Espalhabilidade e toque: A - toque agradável, fácil aplicação na pele; D - toque desagradável, pegajoso; MD - toque muito desagradável, muito pegajoso, compromete a aplicação na pele. Valores expressos em média e desvio padrão (X $\pm \mathrm{DP})$. Teste de Tukey de comparações múltiplas. pH \pm significativamente diferente quando comparado com outras formulações. FPS in vitro \pm significativamente diferentes em relação à formulação 9.

Com base na Tabela 3, pode-se observar que quase todos os fotoprotetores, exceto aqueles que contêm Deanol ${ }^{\circledR} /$ DMAE Fluido (formulações 2 e 7), ambos com e sem fragrância, mostraram valores de $\mathrm{pH}$ com $\mathrm{O}$ intervalo recomendado. Este parâmetro está diretamente envolvido com a qualidade das formulações. Formulações 2 e 7 apresentaram pH significativamente mais baixo do que os outros independentemente da fragrância. Além disso, os valores de $\mathrm{pH}$ encontrados $(3,61 \pm 0,06$ e 3,61 \pm 0,04 , respectivamente) estão abaixo da faixa recomendada para a estabilidade de ambos os filtros UV químicos e Deanol ${ }^{\circledR} /$ DMAE Fluido, indicando que a estabilidade destas formulações pode ser menor do que as outras, comprometendo sua vida útil estimada (SOUZA, 2004; SOUZA, 2005). Isso significa que o pH dos protetores solares compostos contendo Deanol ${ }^{\circledR /} /$ DMAE Fluido deve ser aumentado para 6,0-7,0, garantindo a estabilidade, tanto os filtros UV e químicos e ativos antienvelhecimento.

Finalmente, a Tabela 3 exibe a variação in vitro do FPS. Nenhuma diferença significativa foi encontrada após a adição de agentes ativos antienvelhecimento em fotoprotetores. No entanto, os filtros solares contendo Enteline ${ }^{\circledR} 2$ (Formulações 4 e 9) in vitro, apresentaram no FPS formulações sem fragrância significativamente maior. Isso mostra que a fragrância usada, pode interferir no FPS e, no caso deste agente ativo antienvelhecimento, esta fragrância deve ser evitado para não prejudicar o desempenho do produto. 


\section{CONCLUSÃO}

Em conclusão, pode-se dizer que as preparações estudadas mostraram bastante estabilidade física, embora não tenham sido realizadas nem a longo prazo, nem com estudos de estabilidade acelerada. $\mathrm{O}$ intervalo de $\mathrm{pH}$ de máxima estabilidade foi apresentado de acordo com a necessidade de filtros solares contendo compostos Longevicell ${ }^{\circledR}$, Enteline ${ }^{\circledR} 2$ ou Iris Iso ${ }^{\circledR}$ como agentes ativos antienvelhecimento. No entanto, as formulações contendo Deanol ${ }^{\circledR} /$ DMAE Fluido devem ser ajustadas para uma faixa de $\mathrm{pH}$ 6,0-7,0, independentemente da fragrância. Além disso, a fragrância usada em fotoprotetores contendo Enteline ${ }^{\circledR} 2$ avaliadas neste trabalho pode diminuir significativamente o FPS na preparação e, portanto, devem ser evitada. Finalmente, a demanda por protetores solares contendo agentes ativos antienvelhecimento aumentou, indicando a necessidade do controle de qualidade neste tipo de preparação em farmácias de manipulação, a fim de garantir a eficácia e segurança desses produtos.

\section{REFERÊNCIAS}

Brasil. Ministério da Saúde. Agencia Nacional de Vigilância Sanitária. Guia de Estabilidade de produtos cosméticos. [Online]. Brasília; 2004. [capturado 15 jul. 2010]. Disponível em: http://www.anvisa.gov.br/divulga/public/serie/cos méticos.pdf

Borguetti GS, Knorst MT. Desenvolvimento e avaliação da estabilidade física de loções O/A contendo filtros solares. Rev Bras Cienc Farm 2006;42:531-537.

Fonseca A, Prista LN. Manual de terapêutica dermatologica e cosmetologia. São Paulo: Roca, 2000. 436p.
Gil ES. Controle físico-químico de qualidade de medicamentos. 2.ed. São Paulo: Pharmabooks, 2007, p. 455-457.

Krutmann J, Gilchrest BA. Photoaging of skin. In: Gilchrest BA, Krutmann J, eds. Skin aging. Berlin: Springer, 2006:33-44. http://dx.doi.org/10.1007/3540-32953-6 4

Krutmann J, Yarosh D. Modern photoprotection of human skin. In: Gilchrest BA, Krutmann J, eds. Skin aging. Berlin: Springer, 2006:103-112. http://dx.doi.org/10.1007/3-540-32953-6 9

Longevicell $^{\circledR}$. Informação farmacotécnica [Online] 2010. [capturado 20 jul. 2010]; Disponível em: http://www.galena.com.br/longevicell/hotsite.html

Mansur JS, Breder MNR, Mansur MCA, Azulay RD. Determinação do fator de proteção solar por espectrofotometria. An Bras Dermat 1986; 61:121-124.

Sanfeld A, Steinchen A. Emulsions stability, from dilute to dense emulsions - Role of drops deformation. Adv Colloid Interfac 2008;140: 1-65. http://dx.doi.org/10.1016/j.cis.2007.12.005

Santos EP, Freitas ZM, Souza KR, Garcia S, Vergnanini $A$. In vitro and in vivo determinations of sun protection factors of sunscreen lotions with octylmethoxycinnamate. Int J Cosmetic Sci 1999; 21:1-5. http://dx.doi.org/10.1046/j.14672494.1999.181658.x

Stanfield JW. Sun protectants: enhancing product functionality with sunscreens. In: Schueller R, Romanowski P. Multifunctional cosmetics. New York: Marcel Dekker, 200. p.145-160.

Souza VM. Ativos dermatológicos. 2.ed. São Paulo: Tecnopress, 2004;1:63-81.

Souza VM. Ativos dermatológicos. São Paulo: Tecnopress, 2005; 3:127-130.

Tadros TF. Future developments in cosmetic formulations. Int J Cosm Sci 1992; 14:93-111. 
http://dx.doi.org/10.1111/j.1467-

2494.1992.tb00045.x

Vieira RP, Fernandes AR, Kaneko TM, Consiglieri VO, Pinto CASO, Pereira CSC, Baby AR, Velasco MVR. Physical and physicochemical stability evaluation of cosmetic formulations containing soybean extract fermented by Bifidobacterium animalis. Braz J Pharm Sci 2009; 45: 515-525.

http://dx.doi.org/10.1590/S1984-

$\underline{82502009000300018}$ 